\title{
General Taylor configuration expansion revisited
}

\author{
M. S. Chu, T. H. Jensen, and P. M. Bellan ${ }^{\mathrm{a})}$ \\ General Atomics, P. O. Box 85608, San Diego, California 92186-5608
}

(Received 20 November 1998; accepted 27 January 1999)

The validity of a series expansion proposed previously [T. H. Jensen and M. S. Chu, Phys. Fluids 27, 2881 (1984)] for describing general Taylor configurations of magnetized plasmas has been reexamined because an apparent paradox was realized. From analyses of simple cases which can be dealt with mostly analytically, it is concluded that the paradox is a Gibbs phenomenon, and that the series expansion is valid. (C) 1999 American Institute of Physics. [S1070-664X(99)02005-4]

\section{INTRODUCTION}

From investigation of the relaxation process in a linear pinch, J. B. Taylor ${ }^{1}$ proposed that any magnetically confined, high temperature plasma, surrounded by a closed conducting wall (with no perpendicular magnetic field) will relax toward a configuration of the form

$$
\boldsymbol{\nabla} \times \mathbf{B}+\sigma \mathbf{B}=0,
$$

where $\mathbf{B}$ is the magnetic field strength and $\sigma$ is a constant. Such configurations are commonly called 'Taylor' configurations. Taylor also showed that the configuration is a consequence of the assumption that the magnetic helicity, $K$ $=\int \mathbf{A} \cdot \mathbf{B} d V$, is conserved and the magnetic energy, $E$ $=(1 / 2) \int \mathbf{B}^{2} d V$, is minimized. Here, $\mathbf{A}$ is the magnetic vector potential and $V$ is the volume inside the closed, conducting wall. The relevance of Taylor's assumption to real plasmas has been amply demonstrated. ${ }^{2}$

A formalism for describing Taylor configurations for cases where the shape of the closed, conducting wall is arbitrary and where a "frozen-in," perpendicular magnetic field component at the wall may exist, was given earlier. ${ }^{3}$ Frozen-in fields can exist also without normal field components at the wall when the volume enclosed is not singly connected. The formalism provided convenient ways of viewing methods for helicity injection or current drive into plasmas as well as useful relationships between $K, E$, and $\sigma$. The formalism utilized an expansion in eigenfunctions of the curl operator. An assumption on completeness of these eigenfunctions was made that later was proven correct by Yoshida and Giga. ${ }^{4}$

Recently one of us (P.M.B.) noticed an apparent paradox associated with the formalism for the case of a nonvanishing normal magnetic field at the surrounding wall. For this case there must be a nonvanishing normal current density at the wall according to (1). But, paradoxically, all elements of the formal expansion have vanishing normal components of the current density at the wall. From an analysis of this phenomenon for simple examples, which can be treated mostly analytically, we conclude that the paradox is an example of the Gibbs phenomenon ${ }^{5}$ and that the previously proposed expansion is valid.

${ }^{a}$ California Institute of Technology, Pasadena, California 91125
The layout of the paper is as follows. A brief recapitulation of the formalism of Ref. 3 is given in Sec. II. Section III gives details of the analysis of one of the examples investigated, namely a conducting sphere with a frozen-in field corresponding to a uniform magnetic field. A brief discussion of conclusions is given in Sec. IV.

\section{RECAPITULATION OF THE FORMALISM}

The formalism deals with the problem of describing the Taylor configuration specified by (i) the shape of the closed, conducting wall, (ii) the normal component of the magnetic field at the wall, (iia) in case of a multiple connected wall, locked in magnetic fluxes, and (iii) the total magnetic helicity inside the wall.

The vector potential of the Taylor configuration can be written in the form

$$
\mathbf{A}=\mathbf{A}_{I}+\sum_{\nu} \alpha_{\nu} \mathbf{a}_{\nu}
$$

Here, the inhomogeneous part $\mathbf{A}_{I}$ is associated with the frozen-in fields, in that $\boldsymbol{\nabla} \times \mathbf{A}_{I}$ equals the field that would exist in the absence of plasma currents. Therefore, in the volume inside the wall we have

$$
\boldsymbol{\nabla} \times \boldsymbol{\nabla} \times \mathbf{A}_{I}=0 .
$$

These conditions determine $\mathbf{A}_{I}$ (except for a gauge transformation) when the frozen-in field is given. The vector functions, $\mathbf{a}_{\nu}$ of (2), are the eigenfunctions of ( $\lambda_{\nu}$ 's are the eigenvalues)

$$
\boldsymbol{\nabla} \times \boldsymbol{\nabla} \times \mathbf{a}_{\nu}+\lambda_{\nu} \boldsymbol{\nabla} \times \mathbf{a}_{\nu}=0
$$

with the boundary condition that $\mathbf{a}_{\nu}=0$, and therefore $\boldsymbol{\nabla}$ $\times \mathbf{a}_{\nu} \cdot \hat{n}=0$, at the wall. Here $\hat{n}$ is the unit vector normal to the plasma boundary. We assume the eigenfunctions are complete and prove ${ }^{3}$ that they can be ortho-normalized to satisfy

$$
\int \mathbf{a}_{\mu} \cdot \boldsymbol{\nabla} \times \mathbf{a}_{\nu} d V=-\frac{\lambda_{\nu}}{\left|\lambda_{\nu}\right|} \delta_{\mu \nu} .
$$

It is straightforward to find from (2)-(5) that (1) and the boundary conditions are satisfied when the expansion coefficients, $\alpha_{\nu}$ of (2), are 


$$
\alpha_{\nu}=\sigma \frac{\lambda_{\nu} /\left|\lambda_{\nu}\right|}{\sigma-\lambda_{\nu}} \int \mathbf{a}_{\nu} \cdot \nabla \times \mathbf{A}_{I} d V \equiv \sigma \frac{\lambda_{\nu} /\left|\lambda_{\nu}\right|}{\sigma-\lambda_{\nu}} I_{\nu},
$$

where $I_{\nu}=\int \mathbf{a}_{\nu} \cdot \boldsymbol{\nabla} \times \mathbf{A}_{I} d V$. The apparent paradox is evident from inspection of (2). If we take the $\boldsymbol{\nabla} \times \boldsymbol{\nabla} \times$ of (2), it follows from (3) that the seed field carries no current and from (4) that each of the eigenfunctions $\mathbf{a}_{\nu}$ carries no perpendicular current at the plasma boundary. Using the formalism one can find an expression for the helicity

$$
K=\int \mathbf{A} \cdot \boldsymbol{\nabla} \times \mathbf{A} d V=\sum_{\nu} I_{\nu}^{2} \frac{\lambda_{\nu}}{\left|\lambda_{\nu}\right|}\left[1-\frac{\lambda_{\nu}^{2}}{\left(\sigma-\lambda_{\nu}\right)^{2}}\right]+K_{V},
$$

and for the energy

$$
E=\frac{1}{2} \int\left(\boldsymbol{\nabla} \times \mathbf{A}_{I}\right)^{2} d V=\frac{1}{2} \sum_{\nu} I_{\nu}^{2}\left|\lambda_{\nu}\right| \frac{\sigma^{2}}{\left(\sigma-\lambda_{\nu}\right)^{2}}+E_{V} .
$$

In (7), $K_{V}$ is the "vacuum helicity," $K_{V}=\int \mathbf{A}_{I} \cdot \boldsymbol{\nabla} \times \mathbf{A}_{I} d V$, and $E_{V}$ in (8) is the "vacuum energy," $E_{V}=1 / 2 \int(\boldsymbol{\nabla}$ $\left.\times \mathbf{A}_{I}\right)^{2} d V$. Note that the theory is completely gauge invariant, i.e., $\alpha_{\nu}, I_{\nu}, K$, and $E$ are invariant under the gauge transformation of $\mathbf{A} \rightarrow \mathbf{A}+\boldsymbol{\nabla} \phi$. It follows that for any value of $K$, there is at least one value of $\sigma$ which will satisfy (7). When (7) is satisfied by more than one value of $\sigma$, the Taylor configuration is the one for which the energy is the smallest. It is shown in Ref. 3 that the Taylor configuration is associated with the one and only one value of $\sigma$ larger than the largest negative eigenvalue and smaller than the smallest positive eigenvalue. For the special case that the "coupling coefficient" for one of these eigenvalues vanishes, $I_{\nu_{1}}=0$, the expressions (6)-(8) are not defined for $\sigma=\lambda_{\nu_{1}}$. As discussed in Ref. 3, this ambiguity is resolved by adding an infinitesimal "error field" to $\mathbf{A}_{I}$ so that the $I_{\nu_{1}}$ 's do not vanish. One sees that for the special case $\boldsymbol{\nabla} \times \mathbf{A}_{I}=0, \sigma$ must equal either the largest negative or the smallest positive eigenvalue of (4), depending on the sign of $K$.

\section{EXAMPLE: A SPHERICAL CONDUCTING WALL}

Without any frozen-in field, the case was solved by Rosenbluth and Bussac. ${ }^{6}$ With a uniform "frozen-in" field added, the configuration becomes topologically equal to the "Bumpy Z Pinch",7 or the "Flux Core Spheromaks.",

We employ a spherical coordinate system $(r, \theta, \phi)$. The magnetic field can be represented by the poloidal flux function $\psi$ through

$$
\mathbf{B}=\sigma \psi \nabla \phi+\nabla \phi \times \nabla \psi
$$

From (9) and (1), one gets

$$
r^{2} \boldsymbol{\nabla} \cdot\left(\frac{\boldsymbol{\nabla} \psi}{r^{2}}\right)+\sigma^{2} \psi=0 .
$$

The exact solution to this problem for $\psi$ is

$$
\psi=-\frac{r r_{0} j_{1}(\sigma r)}{2 j_{1}\left(\sigma r_{0}\right)} \sin ^{2} \theta,
$$

and

$$
j_{m}(S)=(\pi / 2 S)^{1 / 2} J_{m+1 / 2}(S),
$$

where $J$ is the ordinary Bessel function. One finds for the exact solution $\mathbf{B}^{E}$ of this problem

$$
\begin{aligned}
& B_{r}^{E}=\frac{r_{0} j_{1}(\sigma r)}{r j_{1}\left(\sigma r_{0}\right)} \cos \theta, \\
& B_{\theta}^{E}=-\frac{r_{0}}{2 j_{1}\left(\sigma r_{0}\right)} \frac{1}{r} \frac{d}{d r}\left[r j_{1}(\sigma r)\right] \sin \theta, \\
& B_{\phi}^{E}=-\frac{\sigma r_{0} j_{1}(\sigma r)}{2 j_{1}\left(\sigma r_{0}\right)} \sin \theta .
\end{aligned}
$$

Here, $r_{0}$ is the radius of the conducting sphere. One sees from (12) that in the absence of a frozen-in field, one must require $j_{1}\left(\sigma r_{0}\right)=0$.

For this case one finds that the components of the eigenfunctions of (4) may be of the form

$$
\begin{aligned}
& a_{\nu r}=b_{\nu}\left[j_{m}\left(\left|\lambda_{\nu}\right| r\right) P_{m}^{n}(\cos \theta) r \cos n \phi\right], \\
& a_{\nu \theta}=b_{\nu}\left[-j_{m}\left(\left|\lambda_{\nu}\right| r\right) P_{m}^{n}(\cos \theta) \frac{n}{\lambda_{\nu} \sin \theta} \sin n \phi\right], \\
& a_{\nu \phi}=b_{\nu}\left[j_{m}\left(\left|\lambda_{\nu}\right| r\right) P_{m}^{n^{\prime}}(\cos \theta) \frac{\sin \theta}{\lambda_{\nu}} \cos (n \phi)\right] .
\end{aligned}
$$

The details of the algebra for the derivation of $\mathbf{a}_{\nu}$ are given in the Appendix. Here the $P$ 's are Legendre's associated functions of the first kind and $P_{m}^{n^{\prime}} \equiv d / d x\left[P_{m}^{n}(x)\right]$. The eigenvalues are determined by

$$
j_{m}\left(\left|\lambda_{\nu}\right| r_{0}\right)=0
$$

Thus for each set of the indices $m$ and $n$ there is a series of $\nu$ 's. The normalization constants $b_{\nu}$ are found to be

$$
\begin{aligned}
b_{\nu}= & \frac{1}{\left|j_{m+1}\left(\left|\lambda_{\nu}\right| r_{0}\right)\right|}\left(\frac{\left|\lambda_{\nu}\right|}{\pi r_{0}^{3}}\right)^{1 / 2} \\
& \times \frac{(2 m+1)^{1 / 2}}{[2 m(m+1)]^{1 / 2}}\left[\frac{(m-n) !}{(m+n) !} \frac{1}{1+\delta_{n 0}}\right]^{1 / 2} .
\end{aligned}
$$

Here, $\delta_{n 0}$ is the Kroenecker symbol.

We consider only the case where the frozen-in field corresponds to a uniform field. Then the coupling coefficients vanish unless $n=0$ and $m=1$. For the $n=0, m=1$ case we can further calculate the coupling coefficients from (6),

$$
I_{\nu}=\frac{4 \pi}{3} b_{\nu} \frac{r_{0}^{3}}{\left|\lambda_{\nu}\right|} j_{2}\left(\left|\lambda_{\nu}\right| r_{0}\right)
$$

and by taking the curl of (2) one can get the series expansion expression for the components of the magnetic field 

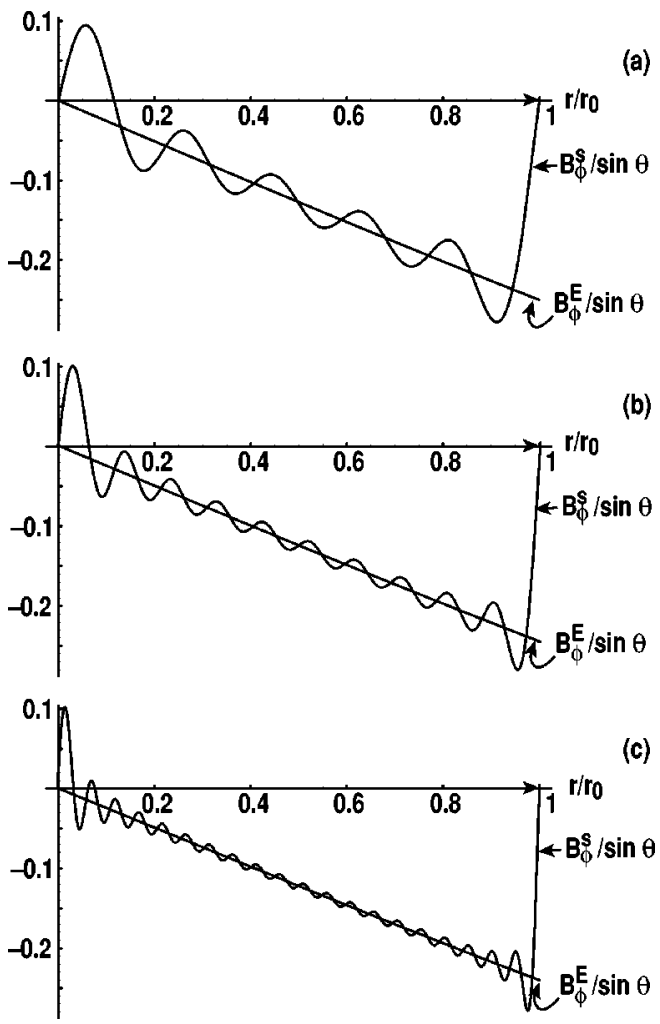

FIG. 1. Comparison of the exact solution $B_{\phi}^{E} / \sin \theta$ given by (12) with the series approximation $B_{\phi}^{s} / \sin \theta$ given in (17) for different number of terms included in the series approximation for a case in which $\sigma r_{0}=0.5$. Ten terms are included in (a), 20 terms in (b), and 40 terms in (c). We note the persistent discrepancy at $r=r_{0}$ and near $r=0$. At other locations, with increasing number of terms included, $B_{\phi}^{s}$ approaches $B_{\phi}^{E}$ with increasing number of oscillations and reduced oscillation amplitudes, i.e., a manifestation of the Gibbs phenomenon.

$$
\begin{aligned}
B_{r}^{s}= & \cos \theta\left[1-\sum_{\lambda_{\nu}>0} \frac{2 \sigma^{2}}{\lambda_{\nu} j_{2}\left(\lambda_{\nu} r_{0}\right)} \frac{1}{\sigma^{2}-\lambda_{\nu}^{2}} \frac{j_{1}\left(\lambda_{\nu} r\right)}{r}\right], \\
B_{\theta}^{s}= & \sin \theta\left\{-1+\sum_{\lambda_{\nu}>0} \frac{\sigma^{2}}{\lambda_{\nu} j_{2}\left(\lambda_{\nu} r_{0}\right)} \frac{1}{\sigma^{2}-\lambda_{\nu}^{2}} \frac{1}{r} \frac{d}{d r}\right. \\
& \left.\times\left[r j_{1}\left(\lambda_{\nu} r\right)\right]\right\}, \\
B_{\phi}^{s}= & \sin \theta\left[\sum_{\lambda_{\nu}>0} \frac{\sigma \lambda_{\nu}}{\sigma^{2}-\lambda_{\nu}^{2}} \frac{j_{1}\left(\lambda_{\nu} r\right)}{j_{2}\left(\lambda_{\nu} r\right)}\right] .
\end{aligned}
$$

Here the notation $\lambda_{\nu}>0$ under the summation symbols means that only terms for which $\lambda_{\nu}>0$ are included.

The exact solution, $B_{r}^{E}, B_{\theta}^{E}$, and $B_{\phi}^{E}$ of Eq. (12) can be compared to the series expansion expression, $B_{r}^{s}, B_{\theta}^{s}, B_{\phi}^{s}$ of Eq. (17). We found that with just a few terms of the series expansion $B_{r}^{s}$ and $B_{\theta}^{s}$ get close to $B_{r}^{E}$ and $E_{\theta}^{E}$, respectively. The discrepancy between $B_{\phi}^{E}$ and $B_{\phi}^{s}$ is more pronounced. In Fig. 1 is shown $B_{\phi}^{E} / \sin \theta$ and $B_{\phi}^{s} / \sin \theta$ versus $r / r_{0}$ from the cases of using 10, 20, and 40 terms in the series expansion. One notices that the series expansion function is approaching the exact function as the number of terms is increased. There is, however, a notable persistent difference at $r / r_{0}=1$, in that the exact function is finite while the series expansion function is zero. This is a manifestation of the paradox; for the
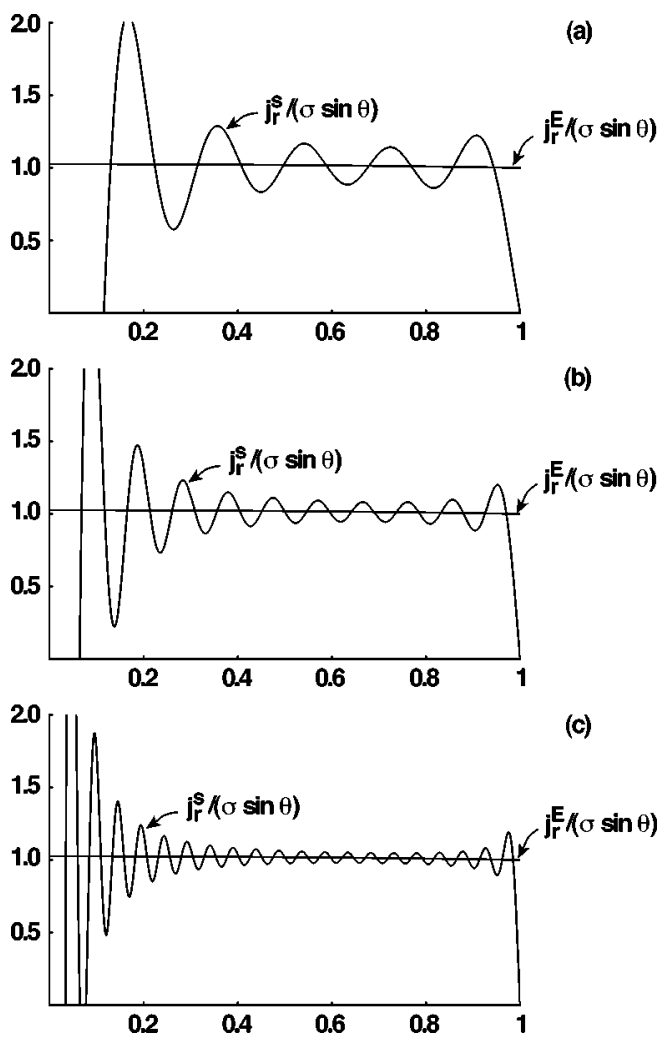

FIG. 2. Comparison of the exact solution $j_{r}^{E} /(\sigma \cos \theta)$ derived from (12) with the series approximation $j_{r}^{s} /(\sigma \cos \theta)$ given by (18) for different number of terms included in the series approximation for a case in which $\sigma r_{0}$ $=0.5$. Ten terms are included in (a), 20 terms in (b), and 40 terms in (c). We note the persistent discrepancy at $r=0$ and $r=r_{0}$. At other locations, with increasing number of terms used in the series solution, $j_{r}^{s}$ approaches $j_{r}^{E}$.

exact solution there is a radial current density where there is a normal component of the magnetic field at the wall, while the series expansion solution has a vanishing normal current density at the wall. This discrepancy exists only in a region near the wall, and with increasing number of terms in the expansion, this region of discrepancy shrinks. It is an example of the Gibbs phenomenon.

Next we examine the series expansion expression for the current. Taking the curl of the expression for $\mathbf{B}^{s}$, given by (17), one obtains

$$
\begin{aligned}
& j_{r}^{s}=-\sigma\left[\sum_{\lambda_{\nu}>0} \frac{\lambda_{\nu}}{j_{2}\left(\lambda_{\nu} r_{0}\right)\left(\sigma^{2}-\lambda_{\nu}^{2}\right)} \frac{2}{r} j_{1}\left(\lambda_{\nu} r\right)\right] \cos \theta \\
& j_{\theta}^{s}=\sigma\left\{\sum_{\lambda_{\nu}>0} \frac{\lambda_{\nu}}{j_{2}\left(\lambda_{\nu} r_{0}\right)\left(\sigma^{2}-\lambda_{\nu}^{2}\right)} \frac{1}{r} \frac{d}{d r}\left[r j_{1}\left(\lambda_{\nu} r\right)\right]\right\} \sin \theta \\
& j_{\phi}^{s}=\sigma\left[\sum_{\lambda_{\nu}>0} \frac{\sigma \lambda_{\nu}}{\sigma^{2}-\lambda_{\nu}^{2}} \frac{j_{1}\left(\lambda_{\nu} r\right)}{j_{2}\left(\lambda_{\nu} r_{0}\right)}\right] \sin \theta
\end{aligned}
$$

The notation on summation used in (17) is also used here. One notices that $j_{\phi}^{s}=\sigma B_{\phi}^{s}$, which is the relationship for the exact solution. One sees also from (17) and (18) that the relationships between $j_{r}^{s}$ and $B_{r}^{s}$ as well as between $j_{\theta}^{s}$ and $B_{\theta}^{s}$ are more complicated. In particular, it is found that $j_{r}^{s} / B_{r}^{s}$ and $j_{\theta}^{s} / B_{\theta}^{s}$ are not equal to the constant value $\sigma$. The fidelity of the series expansion for the current is illustrated in Fig. 2. 

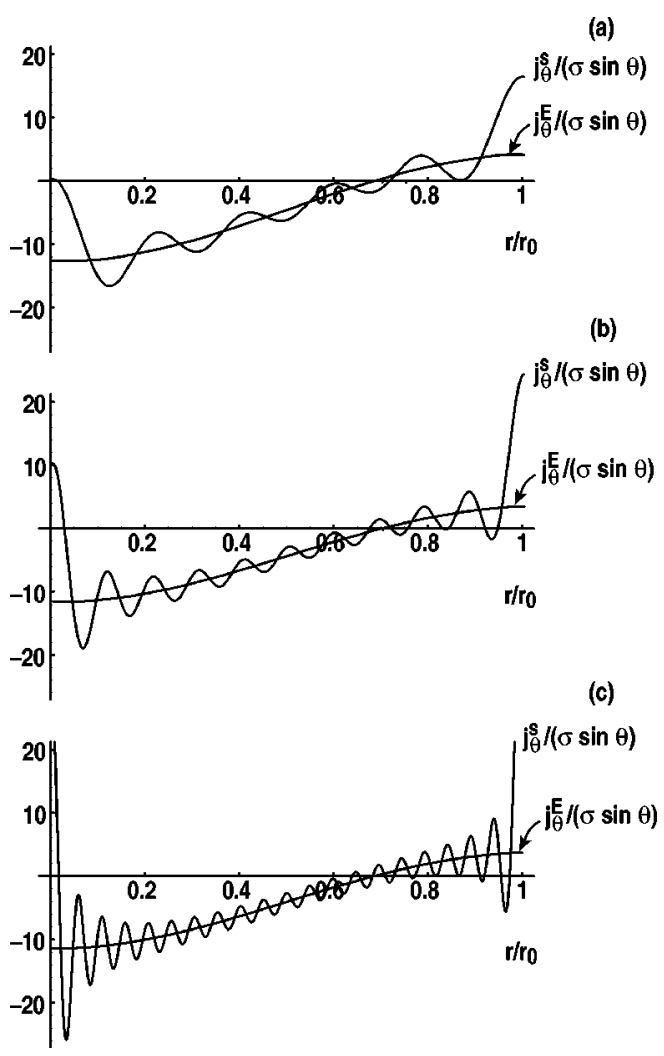

FIG. 3. Comparison of the exact solution $j_{\theta}^{E} /(\sigma \sin \theta)$ derived from (12) with the series approximation $j_{s}^{E} /(\sigma \sin \theta)$ given by (18) for different number of terms included in the series approximation for a case in which $\sigma r_{0}$ $=4$. Ten terms are included in (a), 20 terms in (b), and 40 terms in (c). We note the persistent discrepancy at $r=0$ and $r=r_{0}$. At other locations, with increasing number of terms used in the series solution, $j_{\theta}^{s}$ does not approach $j_{\theta}^{E}$, but oscillates around it with a nondecreasing amplitude. In this case, $j_{\theta}^{s}$ approaches $j_{\theta}^{E}$ only through coarse graining.

Here are shown $j_{r}^{s} /(\sigma \cos \theta)$ versus $r / r_{0}$ as well as $j_{r}^{E} /(\sigma \cos \theta)$ versus $r / r_{0}$, where $\mathbf{j}^{E}=\boldsymbol{\nabla} \times \mathbf{B}^{E} ; \mathbf{B}^{E}$ is given by (12). Here also three cases are displayed, using 10,20, and 40 terms of the series expansion. One recognizes differences between the exact function and that obtained from the series expansion, particularly near $r / r_{0}=0$ and 1 . The series solution $j_{r}^{s}$ always has the value 0 at $r=r_{0}$, whereas the exact solution $j_{r}^{E}$ is nonzero there. Again, as the number of terms increases, $j_{r}^{s}$ approaches $j_{r}^{E}$. An even more interesting case is the difference between $j_{\theta}^{s}$ and $j_{\theta}^{E}$. We found that the amplitude of the difference between the exact and the series expansion functions in this case does not decrease with increasing number of terms in the expansion. They tend to each other only through a coarse graining process. This is shown in Fig. 3. Again we conclude that the difference is an example of the Gibbs phenomenon. ${ }^{5}$

For the case of a spherical conducting wall with a frozen-in field corresponding to a uniform field, we have also calculated the relationships $K(\sigma)-K_{V}$ and $E(\sigma)-E_{V}$ given by (7) and (8). As expected these integral quantities are insensitive to the number of terms in the expansion. In Fig. 4 are shown $K-K_{V}$ and $E-E_{V}$ versus $\sigma$. One notices the important branch of $K(\sigma)-K_{V}$, which is monotonically decreasing from $+\infty$ at the largest of the negative eigenvalues

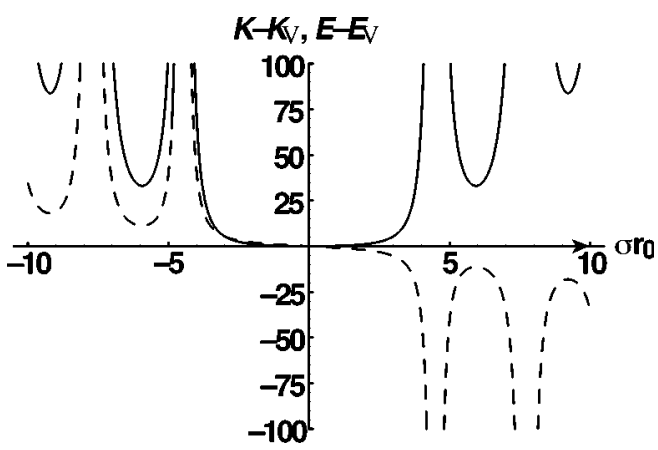

FIG. 4. The dependence of $K-K_{V}$ (dotted line) and $E-E_{V}$ (solid line) in arbitrary units on $\sigma r_{0}$ for the case of a spherical conducting wall and a uniform frozen-in field.

toward $-\infty$ at the smallest positive eigenvalue. One may also see from Fig. 4 (as proven in Ref. 3) that for a given value of $K$, the solution with the lowest energy has a value of $\sigma$ larger than the largest negative eigenvalue and smaller than the smallest positive eigenvalue.

A similar analysis was also made of the case of a conducting circular cross-section cylinder with frozen-in fields corresponding to uniform fields either parallel or perpendicular to the cylinder. The results obtained are similar to those obtained for the case of a conducting sphere, namely, the existence of a boundary layer in which the series solution $\mathbf{B}^{s}$ does not converge to the exact solution $\mathbf{B}^{E}$. Similarly the series solution on $\mathbf{j}^{s}$ converges toward $\mathbf{j}^{E}$ only by coarse graining, another example of the Gibbs phenomenon.

\section{SUMMARY}

A series expansion for Taylor configurations under general circumstances was reexamined because a recently realized paradox made its validity questionable. Examples were presented for which the exact Taylor configuration could be found analytically and for which also the complete set of functions used in the series expansion could be found analytically. For these cases comparisons were made between the exact solutions and those using the series expansion with different numbers of terms. The differences between the exact and the expansion solutions exhibit the characteristics of the Gibbs phenomenon. Specifically, the paradox was that the exact solution has a finite current density normal to the conducting wall wherever there is a normal magnetic field component, while all terms of the expansion have a vanishing normal component of the current density at the wall. As an illustration, consider the case of the plasma enclosed by a thin conducting wall. Where the normal magnetic field is finite, the direction of the current density turns abruptly 90 $\mathrm{deg}$ at the wall. It is this discontinuity which gives rise to the Gibbs phenomenon. The examples studied showed that this discrepancy only exists in a layer near the wall and that the thickness of the layer is approaching zero when the number of terms included in the expansion approaches infinity. Thus we conclude that the series expansion in question is valid. However, because of the Gibbs phenomenon, although the theory is valid, one should use caution in applying the theory. It is readily applicable when global integrated quan- 
tities such as $K$ and $E$ for the configurations are required. But it is not directly applicable when local quantities such as local magnetic field at the plasma boundary or current at a particular point are required.

\section{ACKNOWLEDGMENT}

This work was supported by the U.S. Department of Energy under Grant Nos. DE-FG03-95ER54309 and DE-FG0398ER54461.

\section{APPENDIX A: DERIVATION OF THE VECTOR POTENTIAL FUNCTIONS $a_{v}$ IN SPHERICAL COORDINATE SYSTEM}

The vector potential functions $\mathbf{a}_{\nu}$ satisfy Eq. (4) with the boundary condition $\mathbf{a}_{\nu}=0$,

$$
\boldsymbol{\nabla} \times \boldsymbol{\nabla} \times \mathbf{a}_{\nu}+\lambda_{\nu} \mathbf{a}_{\nu}=0 .
$$

The easiest way to obtain an explicit solution for $\mathbf{a}_{\nu}$ is to start from the solutions given by Rosenbluth and Bussac ${ }^{6}$ for $\mathbf{b}_{\nu}$

$$
\begin{aligned}
b_{\nu r}= & b_{m}^{n} e^{i n \phi}\left[-m(m+1) P_{m}^{n} \frac{j_{m}\left(\left|\lambda_{\nu}\right| r\right)}{\lambda_{\nu} r}\right] \\
b_{\nu \theta}= & b_{m}^{n} e^{i n \phi}\left[\frac{i n}{\sin \theta} P_{m}^{n}+\sin \theta P_{m}^{n^{\prime}} \frac{1}{\lambda_{\nu} r} \frac{d}{d r} r j_{m}\left(\left|\lambda_{\nu}\right| r\right)\right], \\
b_{\nu \phi}= & b_{m}^{n} e^{i n \phi}\left[\sin \theta P_{m}^{n^{\prime}}(\cos \theta) j_{m}\left(\left|\lambda_{\nu}\right| r\right)\right. \\
& \left.-\frac{i n}{\sin \theta} P_{m}^{n} \frac{1}{\lambda_{\nu} r} \frac{d}{d r}\left(r j_{m}\right)\right]
\end{aligned}
$$

with the boundary condition of $j_{m}\left(\left|\lambda_{\nu}\right| r\right)=0$. Note that with this choice for the boundary condition $b_{\nu r}=0$ but $b_{\nu \phi}$ and $b_{\nu \phi}$ remain finite at $r=r_{0}$. However, $\mathbf{a}_{\nu}$ is related to $\mathbf{b}_{\nu}$ by a gauge transformation

$$
\mathbf{a}_{\nu}=\mathbf{b}_{\nu}+\nabla \Phi \text {. }
$$

In order for $\mathbf{a}_{\nu}$ to satisfy its boundary condition, it is necessary for the components of $\nabla \Phi$ to behave as

$$
\begin{aligned}
& \frac{\partial \Phi}{\partial r}=0, \\
& \frac{1}{r} \frac{\partial \Phi}{\partial \theta}=-b_{m}^{n} e^{i n \phi} \sin \theta P_{m}^{n^{\prime}} \frac{1}{\lambda_{\nu} r} \frac{d}{d r} r j_{m}\left(\left|\lambda_{\nu}\right| r\right), \\
& \frac{1}{r \sin \theta \partial \phi}=b_{m}^{n} e^{i n \phi} \frac{i n}{\sin \theta} P_{m}^{n^{\prime}} \frac{1}{\lambda_{\nu} r} \frac{d}{d r} r j_{m}\left(\left|\lambda_{\nu}\right| r\right) .
\end{aligned}
$$

The $\theta$ and $\phi$ components of (A4) allow a solution for $\Phi$ as

$$
\Phi=b_{m}^{n} e^{i n \phi} P_{m}^{n} \frac{1}{\lambda_{\nu} r} \frac{d}{d r} r j_{m}\left(\left|\lambda_{\nu}\right| r\right) .
$$

Because $j_{m}$ satisfy the equation

$$
\frac{1}{r} \frac{d^{2}}{d r^{2}}\left(r j_{m}\right)-\frac{m(m+1)}{r^{2}} j_{m}+\lambda_{\nu}^{2} j_{m}=0,
$$

the radial derivative of (A5) will also satisfy the boundary condition (A4) at the plasma boundary. Substitution of (A5) into (A3) gives for the real component of $a_{\nu}$ :

$$
\begin{aligned}
& a_{\nu r}=b_{\nu}\left[j_{m}\left(\left|\lambda_{\nu}\right| r\right) P_{m}^{n}(\cos \theta) r \cos n \phi\right], \\
& a_{\nu \theta}=b_{\nu}\left[-j_{m}\left(\left|\lambda_{\nu}\right| r\right) P_{m}^{n}(\cos \theta) \frac{n}{\lambda_{\nu} \sin \theta} \sin n \phi\right], \\
& a_{\nu \phi}=b_{\nu}\left[j_{m}\left(\left|\lambda_{\nu}\right| r\right) \frac{P_{m}^{n^{\prime}}(\cos \theta)}{\lambda_{\nu}} \sin \theta \cos (n \phi)\right] .
\end{aligned}
$$

In (A7) we have renormalized the amplitude of $\mathbf{a}_{\nu}$ by $\lambda_{\nu}$.

${ }^{1}$ J. B. Taylor, Phys. Rev. Lett. 33, 1139 (1974).

${ }^{2}$ J. B. Taylor, Rev. Mod. Phys. 58, 741 (1986).

${ }^{3}$ T. H. Jensen and M. S. Chu, Phys. Fluids 27, 2881 (1984).

${ }^{4}$ Z. Yoshida and Y. Giga, Math. Z. 204, 235 (1990).

${ }^{5} \mathrm{M}$. Morse and H. Feshbach, in Methods of Theoretical Physics (McGrawHill, New York, 1953), p. 745.

${ }^{6}$ M. N. Rosenbluth and M. N. Bussac, Nucl. Fusion 19, 489 (1979).

${ }^{7}$ T. H. Jensen and M. S. Chu, J. Plasma Phys. 25, 459 (1981). 